\title{
Recurrent triploidy of maternal origin
}

\author{
Francesco Brancati ${ }^{1,2}$, Rita Mingarelli ${ }^{2}$ and Bruno Dallapiccola*,1,2 \\ ${ }^{1}$ CSS Hospital, IRCCS, San Giovanni Rotondo and CSS-Mendel Institute, Rome, Italy; ${ }^{2}$ Department of Experimental \\ Medicine and Pathology, University 'La Sapienza', Rome
}

Triploidy is the most frequent chromosome aberration in first trimester spontaneous abortions. In contrast to aneuploidies due to nondisjunction, increased maternal age is not a risk factor and the mechanism of triploidy remains poorly understood. To date, recurrence of triploidy of maternal origin has been described only in a few families suggesting some underlying genetic factors. Here, we report on a woman who underwent three consecutive triploid pregnancies, in two of which maternal origin of triploidy was proved by molecular analysis.

European Journal of Human Genetics (2003) 11, 972-974. doi:10.1038/sj.ejhg.5201076

Published online 24 September 2003

Keywords: triploidy; digyny; recurrent abortion

\section{Introduction}

Triploidy occurs approximately in $2 \%$ of conceptuses, and is the most common chromosome aberration in first trimester spontaneous abortions. It can be seen also in premature, stillborn and full-term liveborn infants. ${ }^{1}$ Digyny is the predominant mechanism both in prenatally and postnatally detected cases, possibly due to the longer survival of fetuses with maternally derived triploidy. ${ }^{2,3}$ Different parental origin also accounts for different outcome, digyny being associated with more severe growth retardation, relative macrocephaly and small, nonhydatiform or only partly hydatiform placentas. ${ }^{2,4}$ Unlike most chromosomal anomalies, increased maternal age is not a risk factor for maternal triploidy, and no other susceptibility factor has been identified. Thus, the mechanisms underlying digyny are still unclear. Interestingly, recurrence of molar diandric pregnancies in a few families has proved to be inherited as an autosomal recessive trait and a susceptibility locus assigned to chromosome 19.,6 Here, we report on the recurrence of triploidy in three consecutive pregnancies. Molecular analysis in two of them has shown a maternal origin of the extra set of chromosomes. This observation corroborates two previous reports

*Correspondence: Prof. B Dallapiccola, CSS Mendel Institute, Viale Regina Margherita, 261, 00198 Rome, Italy. Tel: + 39-06-44160536; Fax: + 39-0644160548; E-mail: f.brancati@css-mendel.it

Received 20 February 2003; revised 17 April 2003; accepted 26 June 2003 of recurrent maternally inherited triploidies and argues for an underlying genetic mechanism. ${ }^{7,8}$

\footnotetext{
Case report

Clinical history

A 36-year-old woman was referred for genetic counselling because of three consecutive triploid pregnancies. Family history was unremarkable, with no evidence of consanguinity or multiple miscarriages. She had her first pregnancy at 33 years of age, which was miscarried at 12 weeks of gestation. Chromosome analysis of the abortion revealed a 69, XXY karyotype. The second pregnancy occurred 1 year later. Ultrasound examination at 21 weeks of gestation detected severe oligohydramnios, an Arnold-Chiari type II malformation, with severe hydrocephaly, asymmetric lateral ventricles, porencephaly, lumbosacral myelomeningocele, and atrio-ventricular canal defect, with unilateral hydrothorax. Biparietal diameter (BPD) was $4.5 \mathrm{~cm}$ (mean for gestational age $5.2 \mathrm{~cm}$ ) and femur length (FL) $2.2 \mathrm{~cm}$ (mean for gestational age $3.1 \mathrm{~cm}$ ). An amniocentesis disclosed a 69,XXX karyotype. Spontaneous fetal demise occurred at 24 weeks of gestation. The proband had her third pregnancy at the age of 35 years. While ultrasound scans at 8 weeks of gestation were unremarkable, at 14 weeks reduced amniotic fluid was evident, with $2.8 \mathrm{~cm}$ BPD ( 2 SD below the mean) and $1.02 \mathrm{~cm} \mathrm{FL}(<2 \mathrm{SD}$ below the
} 
Table 1 Microsatellite markers analysis in two triploid fetuses and their parents

\begin{tabular}{|c|c|c|c|c|c|}
\hline Markers & Map position & Mother & Father & Second fetus & Third fetus \\
\hline D2S2271 & $2 q 21$ & $139 / 145$ & $143 / 145$ & $139 / 145$ & $139 / 143$ \\
\hline D4S1627 & $4 p 13$ & $198 / 202$ & $200 / 208$ & $198 / 202 / 208$ & $198 / 202 / 200$ \\
\hline D6S430 & $6 q 12$ & $230 / 234$ & $230 / 232$ & $234 / 230$ & $234 / 230$ \\
\hline D7S663 & $7 q 11$ & $156 / 160$ & $160 / 168$ & $156 / 160$ & $156 / 160$ \\
\hline D8S166 & $8 q 12$ & $110 / 112$ & $114 / 118$ & $110 / 112 / 118$ & $110 / 112 / 118$ \\
\hline D11S922 & $11 \mathrm{p} 15$ & $148 / 152$ & $140 / 152$ & $148 / 152 / 140$ & $148 / 152$ \\
\hline D15S128 & $15 q 11$ & $190 / 198$ & $192 / 192$ & $190 / 198 / 192$ & $190 / 198 / 192$ \\
\hline D18S452 & $18 \mathrm{p} 11$ & $126 / 128$ & $126 / 132$ & $128 / 126$ & $126 / 128 / 132$ \\
\hline
\end{tabular}

Allele sizes are in base pair. Maternal alleles are typed in bold. Markers supporting maternal meiotic origin of triploidy based on presence of three different alleles in both fetuses are underlined.

mean), reflecting an asymmetrical IUGR. The cerebellum could not be visualized and a 'golf ball' image was found in the heart. Karyotype was $69, \mathrm{XXY}$ and pregnancy was terminated at 18 weeks of gestation.

\section{Molecular investigation}

In order to understand the parental origin of repeated triploidies, fetal DNA was obtained from specimens of the second and third abortions. Blood samples were collected from mother and her husband and DNA extracted using standard procedures. A number of highly informative microsatellite markers were selected from the genetic map of the Centre for Medical Genetics, Marshfield Medical Research Foundation (www.marshfieldclinic.org/ research/genetics/). All markers were PCR-amplified using fluorescent-labelled primers, fragments were run on an ABI Prism 3100 DNA sequencer (Applied Biosystems, Foster City, CA, USA), and analysed with GeneScan and Genotyper software (Applied Biosystems, Foster City, CA, USA). Three markers gave uninformative results being homozygous in fetuses and in their mother or in both parents. Eight out of 11 microsatellite markers showed at least two different alleles in both analysed fetuses and their mother. For these markers, fetal and parental genotypes were compared (Table 1). Two distinct maternal and one paternal alleles were observed in both fetuses in three out of eight informative markers. Two additional markers showed a different pattern in the two fetuses with three distinguishable alleles in one fetus and only two in the other. Finally, three markers showed only two distinguishable alleles in both fetuses, one of which was shared by parents. Taken together, these results suggested a maternal origin of triploidy in both fetuses, but the time and mode of formation could not be definitely ascertained. In fact, while markers presenting three distinct fetal alleles are suggestive of an error in maternal meiosis I, microsatellite markers with only two distinguishable alleles are compatible with an error in either maternal meiosis I or II. A semiquantitative evaluation of these markers did not give informative results due to insufficient dosage differences in the two peak profiles. This was probably due to poor quality of fetal DNA samples and nonstringent conditions of PCR amplification. ${ }^{9}$

\section{Discussion}

We describe a woman who underwent three consecutive triploid pregnancies. Cytogenetic analyses revealed, respectively, 69,XXY, 69,XXX and 69,XXY karyotypes. The origin of triploidy was investigated in the last two pregnancies using molecular markers that showed the presence of a maternal extra set of chromosomes. Fetal phenotypes were consistent with digyny in both cases, based on IUGR, oligohydramnios and long survival. ${ }^{4}$

To our knowledge, this is the third report of recurrent triploidy of proved maternal origin. Familial digyny was reported in a woman referred for in vitro fertilization (IVF) because of two previous triploid pregnancies. IVF of 13 embryos resulted in two additional triploid embryos, with an overall number of four triploid conceptuses in this woman. The most likely mechanism event was considered failure of maternal meiosis II. $^{7}$ A second family with recurrent triploidy was reported by Huang et $a l^{8}$ who investigated a 35-year-old woman with three consecutive triploidy pregnancies. In the last pregnancy, molecular analysis proved the maternal origin of triploidy. In the present report, we have shown that the two fetuses available for molecular analysis inherited the extra set of chromosomes from their mother. A mitotic duplication of the maternal pronucleus was ruled out based on the observation of several markers showing three distinguishable fetal alleles. Assessment of the time and mode of triploidy formation was not fully conclusive, the results being consistent both with meiosis I and II error. A comparison between the results obtained by analysis of microsatellites close to the centromere and distal markers localised on either side of the same chromosome would have been helpful. However, the insufficiency of fetal DNA samples did not allow to analyse additional microsatellites. We were also unable to perform a quantitative evaluation of those markers showing only two distinct alleles in the fetuses. Therefore, the maternal origin of triploidy was established in both fetuses, although it was not possible to 
distinguish between a first or second division error. Parental origin of 25 unrelated triploid pregnancies has been investigated by Baumer et al who found maternal origin in 20 cases and paternal origin in five. Comparable frequency of meiosis I and meiosis II errors were observed, but the high rate of meiotic crossovers during oogenesis made this distinction difficult in some digynic cases and weakened any definite conclusion. ${ }^{10}$ It is likely that, in cases of recurrent digynic triploidy, a unique mechanism is accounting for this anomaly, which is possibly related to some inherited mechanism affecting oogenesis. Furthermore, the frequency of recurrent digyny could be underestimated because spontaneous abortion can arise before the time of amniocentesis and karyotype assessment.

\section{References}

1 Schinzel A: Triploidy; in Schinzel A (eds). Catalogue of unbalanced chromosome aberrations in man, 2nd edn. Berlin-New York: Walter de Gruyter, 2001, pp 887-893.

2 Jacobs PA, Szulman AE, Funkhouser J, Matsuura JS, Wilson CC: Human triploidy: relationship between parental origin of the additional haploid complement and development of partial hydatidiform mole. Ann Hum Genet 1982; 46 (Part 3): 223-231.

3 Miny P, Koppers B, Dworniczak B, Bogdanova N et al: Parenta origin of the extra haploid chromosome set in triploidies diagnosed prenatally. Am J Med Genet 1995; 22: 102-106.

4 McFadden DE, Kalousek DK: Two different phenotypes of fetuses with chromosomal triploidy: correlation with parental origin of the extra haploid set. J Med Genet 1991; 15: 535-538.

5 Moglabey YB, Kircheisen R, Seoud M, El Mogharbel N, Van den Veyver I, Slim R: Genetic mapping of a maternal locus responsible for familial hydatiform moles. Hum Mol Genet 1999; 8: 667-671.

6 Sensi A, Gualandi F, Pittalis MC, Calabrese O et al: Mole maker phenotype: possible narrowing of the candidate region. Eur J Hum Genet 2000; 8: 641-644.

7 Pergament E, Confino E, Zhang JX, Roscetti L, Xien Chen P, Wellman D: Recurrent triploidy of maternal origin. Prenat Diagn 2000; 20: 561-563.

8 Huang B, Prensky L, Thangavelu M, Wang S: Three consecutive triploidy pregnancies in a 35-year-old woman: genetic predisposition. Am J Hum Genet 2002; 71 (Suppl): Abstract 2283.

9 Walsh PS, Erlich HA, Higuchi R: Preferential PCR amplification of alleles: mechanisms and solutions. PCR Methods Appl 1992; 1: 241-250.

10 Baumer A, Balmer D, Binkert F, Schinzel A: Parental origin and mechanisms of formation of triploidy: a study of 25 cases. Eur J Hum Genet 2000; 8: 911-917. 